\section{Nuclear Medicine Radiation Risks}

TO THE EDITOR: In recent times, there has been concern about the harmful effects of radiation from diagnostic procedures such as CT. The BEIR VII report (1) invoked data, largely but not entirely from atomic bomb survivors, to support the linear-nothreshold model of radiation-induced cancer down to the levels that are relevant to such procedures. Brenner and Hall (2) elaborated with estimates relating to the risk of CT. Presumably, these principles are also applicable to both diagnostic and therapeutic nuclear medicine.

Two recent articles in The Journal of Nuclear Medicine, by Boreham and Dolling (3) and by Stabin (4) express contrary views, questioning the linear-no-threshold model. Boreham and Dolling (3) even suggest that low levels of radiation are beneficial. Evidently, there is a controversy. I am not writing to take sides, although I must add that I find Stabin's (4) statement, "the benefits of the study...always substantially outweigh [the] risks," simplistic. In any case, the issues are important for nuclear medicine, having a bearing on selectivity for procedures, radiopharmaceutical doses used, and the information regarding risk that is given to patients and subjects in the contexts of both clinical service and research.

I hope that the SNM and The Journal of Nuclear Medicine will foster evenhanded dialog on this subject.

\section{REFERENCES}

1. National Research Council of the National Academies. Health Risks from Exposure to Low Levels of Ionizing Radiation: BEIR VII, Phase 2. Washington, DC: The National Academies Press; 2006.

2. Brenner DJ, Hall EJ. Computed tomography: an increasing source of radiation exposure. N Engl J Med. 2007;357:2277-2284.

3. Boreham DR, Dolling J-A. Risk associated with therapeutic ${ }^{131} \mathrm{I}$ radiation exposure. J Nucl Med. 2008;49:691-693.

4. Stabin MG. Radiopharmaceuticals for nuclear cardiology: radiation dosimetry, uncertainties, and risk. J Nucl Med. 2008;49:1555-1563.

\section{Herbert A. Klein Veterans Affairs Pittsburgh Healthcare System Pittsburgh, Pennsylvania}

DOI: 10.2967/jnumed.108.058099

REPLY: All reasonable measures of course should be taken to minimize patient exposure without compromising the clinical value of any medical procedure. We know, however, that significant benefits are afforded to individual subjects who are exposed to radiation in the course of diagnostic medical procedures and that the associated risks are small, even if we accept the tenets of the linearno-threshold model. Pat Zanzonico has now presented us with numeric estimates of the benefits of several medical studies. These data were presented at the 2007 ASNC meeting, and are currently posted at a Web site of the Health Physics Society (1). The complete analysis is to be published soon.

COPYRIGHT @ 2009 by the Society of Nuclear Medicine, Inc.

\section{REFERENCE}

1. Zanzonico P. Benefits of Medical Radiation Exposures. Health Physics Society Web site. Available at: http://hps.org/hpspublications/articles/Benefitsofmedradexposures. html. Accessed January 7, 2009.

Michael G. Stabin Vanderbilt University Nashville, Tennessee

DOI: 10.2967/jnumed.108.058222

\section{Characterization of Hilar and Mediastinal Foci on ${ }^{18}$ F-FDG PET: New Variables}

TO THE EDITOR: The article by Karam et al. (1) on the features of ${ }^{18}$ F-FDG-concentrating bilateral hilar foci associated with benign or malignant pathologies draws the reader's attention to an important and relevant issue of current PET practice in oncology. The need to develop a logical decision-making algorithm in this confounding situation and educate the interpreting physicians about such an approach to eliminate errors can hardly be overemphasized. I would like to congratulate the authors for their well-done analysis that attempts a solution to this common diagnostic dilemma. Despite significant recent progress in PET technology (e.g., emergence of fusion PET/CT and novel quantitative approaches), accurate characterization of ${ }^{18} \mathrm{~F}-\mathrm{FDG}$-concentrating mediastinal and hilar nodes continues to pose a major diagnostic challenge worldwide. This challenge also has geographic relevance and is of major concern in Asian countries, including India, where tuberculosis has a high prevalence, and hence, the possibility of encountering falsepositive ${ }^{18}$ F-FDG PET lesions is higher than in the West. Tuberculous lesions can demonstrate variable ${ }^{18} \mathrm{~F}-\mathrm{FDG}$ uptake as determined by inflammatory activity $(2-5) .{ }^{18} \mathrm{~F}$-FDG PET is being investigated for its potential in the assessment of disease activity in a wide array of infectious and aseptic inflammatory conditions $(5,6)$. Hence, scientific analyses of the patient data of the past 2 decades are much needed. Analysis of data that can give important clues about the diagnosis will be of help in devising an optimal approach, ultimately obviating invasive biopsies and reducing patient anxiety.

As the authors indicate (1), their study probably represents the first attempt to scientifically correlate multiple PET variables with ultimate outcome. The results of their retrospective analysis reaffirm certain traditional notions about the significance of variables such as symmetry, maximum standardized uptake value, node size on CT, and stability of ${ }^{18} \mathrm{~F}$-FDG uptake during the course of the disease-variables that are being followed in several PET centers in various countries. All these characteristics are important and performed as expected in determining the nature of the lesions in the study of Karam et al. In addition, the results underscore the importance of the absence or presence of ${ }^{18} \mathrm{~F}-\mathrm{FDG}$-avid foci in nonhilar mediastinal nodes, a variable (which they term the "purity" of the lesion) that was found to be an independent determinant after multivariate analysis. Seventy-nine percent of impure scans, versus $18 \%$ of pure scans, represented malignancy in the examined population. Also, the significant dependence of the nature of the 
lesions on the primary malignancy is important to note $(75 \%$ of patients with colorectal carcinoma were found to harbor benign lesions, whereas the corresponding figures for patients with breast carcinoma, lymphoma, and other malignancies were $34 \%, 49 \%$, and $37 \%$, respectively). Such retrospective analyses from other centers, especially those from the other parts of the world, are urgently required, as new PET variables that are important for clinical decision making in this setting may arise. It will be important to observe the impact of these new variables in multicenter analyses. If the impact is confirmed, the results will be a firm basis for developing an algorithm for the interpretation of ${ }^{18} \mathrm{~F}-\mathrm{FDG}$ PET images. A separate analysis along similar lines in patients with lung carcinoma will also be of considerable value in clinical decisions about this important malignancy.

\section{REFERENCES}

1. Karam M, Roberts-Klein S, Shet N, Chang J, Feustel P. Bilateral hilar foci on ${ }^{18} \mathrm{~F}-$ FDG PET scan in patients without lung cancer: variables associated with benign and malignant etiology. J Nucl Med. 2008;49:1429-1436.

2. Paci M, Cavazza A, Ferrari G, Filice A, Sgarbi G. Image of the month: pulmonary tuberculosis mimicking lung metastasis. J Thorac Oncol. 2008;3:660-661.

3. Chen YK, Shen YY, Kao CH. Abnormal FDG-PET imaging in tuberculosis appearing like lymphoma. Clin Nucl Med. 2004;29:124.

4. Yang CM, Hsu CH, Lee CM, Wang FC. Intense uptake of [F-18]-fluoro-2 deoxyD-glucose in active pulmonary tuberculosis. Ann Nucl Med. 2003;17:407-410.

5. Basu S, Chryssikos T, Moghadam-Kia S, Zhuang H, Torigian D, Alavi A. Positron emission tomography as a diagnostic tool in infection: present role and future possibilities. Semin Nucl Med. 2009;39:36-51.

6. Basu S, Zhuang H, Torigian D, Rosenbaum J, Chen W, Alavi A. Functional imaging of inflammatory diseases using nuclear medicine techniques. Semin Nucl Med. In press.

\section{Sandip Basu \\ Radiation Medicine Centre (BARC) Tata Memorial Centre Annexe \\ Bombay, India}

DOI: 10.2967/jnumed.108.059683

\section{${ }^{18}$ F-FDG and ${ }^{18}$ F-FLT Do Not Discriminate Between Reactive and Metastatic Lymph Nodes in Oral Cancer}

TO THE EDITOR: With great interest, we read the recent publication by Nakagawa et al. (1). The authors studied enlarged, nonmetastatic cervical lymph nodes in oral cancer patients. The maximum standardized uptake value of the preoperatively performed ${ }^{18} \mathrm{~F}-\mathrm{FDG}$ PET/CT was calculated, and the lymph node sections were immunohistochemically stained for glucose transporter type 1 (GLUT-1). The authors reported a positive correlation between the maximum standardized uptake value and both the number of secondary follicles and the reactivity index. Furthermore, the immunohistochemical staining pattern for GLUT-1 was markedly similar to the distribution of follicular dendritic cells and in secondary follicles was relatively localized in germinal centers. Therefore, the authors concluded that ${ }^{18} \mathrm{~F}-\mathrm{FDG}$-avid follicular dendritic cells might be the cause of ${ }^{18} \mathrm{~F}$-FDG uptake in reactive cervical lymph nodes, resulting in false-positive reading of ${ }^{18} \mathrm{~F}$-FDG PET images.

To our knowledge, the study by Nakagawa et al. (1) was the first assessing in detail the histologic basis of a positive ${ }^{18} \mathrm{~F}$-FDG PET signal in reactive cervical lymph nodes. One question that arises is whether this observation is typical of oral cavity tumors. The oral cavity harbors a variety of nonpathogenic and (potentially) pathogenic microorganisms that can invade the body when the mucosal barrier is disrupted by an ulcerating tumor. This may thus cause reactive lymphadenopathy of first-echelon lymph nodes. In a similar study, Chung et al. (2) demonstrated GLUT-1 staining and positive ${ }^{18} \mathrm{~F}$-FDG uptake in mediastinal hyperplastic lymph nodes in patients with non-small cell lung cancer. This finding suggests that the phenomenon occurs also in other tumors of epithelial origin.

The second question is whether this limitation of ${ }^{18} \mathrm{~F}-\mathrm{FDG}$ can be solved with other PET tracers. In a previous issue of The Journal of Nuclear Medicine, we reported the role of $3^{\prime}$-deoxy- $3^{\prime}-{ }^{18} \mathrm{~F}$ fluorothymidine $\left({ }^{18} \mathrm{~F}\right.$-FLT $)$ in the detection of cervical lymph node metastases in patients with head and neck cancer (3). We correlated ${ }^{18}$ F-FLT uptake with immunohistochemical assessment of the proliferation markers Ki-67 and iododeoxyuridine. The sensitivity and specificity of ${ }^{18} \mathrm{~F}$-FLT PET for the detection of metastatic nodes were $100 \%$ and $40 \%$, respectively. Labeling indices for Ki-67 and iododeoxyuridine were higher in the germinal centers harboring B-lymphocytes than in the metastatic deposits. Furthermore, the median number of germinal centers per lymph node and the absolute area occupied by germinal centers were significantly higher in the nonmetastatic (reactive) lymph nodes than in the negative lymph nodes. Therefore, it is likely that the active proliferation of B-lymphocytes as detected by $\mathrm{Ki}-67$ and iododeoxyuridine staining was responsible for the ${ }^{18}$ F-FLT uptake and may lead to falsepositive reading of PET images. These findings lead us to conclude that ${ }^{18} \mathrm{~F}$-FLT PET is not useful for differentiating reactive lymph nodes from cervical lymph node metastases in head and neck squamous cell carcinoma. Several groups validated ${ }^{18}$ F-FLT PET for other tumor sites, such as lung and breast, and reported varying numbers for sensitivity and specificity. For the detection of axillary lymph nodes in breast cancer, Smyczek-Gargya et al. (4) found a promising sensitivity and specificity of $87.5 \%$ and $100 \%$, respectively, whereas Been et al. reported a disappointing low sensitivity of $28.5 \%$, with a specificity of $100 \%$ (5). Three groups studied ${ }^{18} \mathrm{~F}$-FLT PET for the detection of mediastinal lymph node metastases in thoracic tumors and also reported a poor sensitivity of $33.3 \%-57 \%$, with a specificity of $93 \%-100 \%(6-8)$.

In summary, reactive lymphadenopathy may be a cause of falsepositive PET readings in head and neck cancer and non-small cell lung cancer and possibly also other epithelial neoplasms. The reasons are ${ }^{18} \mathrm{~F}-\mathrm{FDG}$ uptake by follicular dendritic cells and ${ }^{18} \mathrm{~F}$ FLT uptake by proliferating lymphoid cells in germinal centers of reactive lymph nodes. Clinicians should be aware of this pitfall and the potential consequences for selection and planning of treatment.

\section{REFERENCES}

1. Nakagawa Y, Yamada M, Suzuki Y. ${ }^{18} \mathrm{~F}-\mathrm{FDG}$ uptake in reactive neck lymph nodes of oral cancer: relationship to lymphoid follicles. J Nucl Med. 2008;49:1053-1059.

2. Chung JH, Cho KJ, Lee SS, et al. Overexpression of Glut1 in lymphoid follicles correlates with false-positive ${ }^{18} \mathrm{~F}$-FDG PET results in lung cancer staging. $\mathrm{J} \mathrm{Nucl}$ Med. 2004;45:999-1003.

3. Troost EG, Vogel WV, Merkx MA, et al. ${ }^{18}$ F-FLT PET does not discriminate between reactive and metastatic lymph nodes in primary head and neck cancer patients. J Nucl Med. 2007;48:726-735.

4. Smyczek-Gargya B, Fersis N, Dittmann H, et al. PET with $\left[{ }^{18} \mathrm{~F}\right]$ fluorothymidine for imaging of primary breast cancer: a pilot study. Eur J Nucl Med Mol Imaging. 2004;31:720-724.

5. Been LB, Elsinga PH, de Vries J, et al. Positron emission tomography in patients with breast cancer using ${ }^{18} \mathrm{~F}-3^{\prime}$-deoxy-3'-fluoro-1-thymidine $\left({ }^{18} \mathrm{~F}-\mathrm{FLT}\right)$ : a pilot study. Eur J Surg Oncol. 2006;32:39-43. 